Elizabeth Reyna Luque Flores. ${ }^{1}$

Maria del Carmen Saravia Cardozo. ${ }^{2}$

Carmen Ximena Ortuño Numbela. ${ }^{3}$

Dra. Lizet Jenny Quispe Arancibia. ${ }^{4}$

Tania Mariel Teran

Alvarez. ${ }^{5}$

Julieta Gomez Terrazas. ${ }^{6}$

Correspondencia a:

${ }^{1}$ Médico Familiar. Docente de Embriología y Genética Universidad del Valle UNIVALLE.

${ }^{2}$ Médico Cirujano. Magister en Educación Superior en Salud. Docente de Anatomía de Universidad Mayor de San Simón, Universidad del Valle UNIVALLE, Universidad Franz Tamayo UNIFRANZ y La Universidad de Aquino Bolivia UDABOL.

${ }^{3}$ Médico Cirujano. Docente de Anatomía Mención en Plastinación y Técnicas Anatómicas.

${ }^{4}$ Médico Cirujano. Docente Anatomía humana Universidad Técnica Privada Cosmos UNITEPC

${ }^{5}$ Licenciada lingüística aplicada a la enseñanza de lenguas,

${ }^{6}$ Licenciada en Enfermería. Jefe de prácticas de la carrera de Enfermería UNITEPC.

Cochabamba - Bolivia.

Email de contacto:

elizabeth.luque.flores@ gmail.com

julietagomezterrazas@ gmail.com

Procedencia y arbitraje:

No comisionado, sometido a arbitraje externo

Recibido para publicación: 18 de enero del 2020 Aceptado para publicación: 25 de febrero del 2020

Citar como:

$\mathrm{Re} \mathrm{Ci} \mathrm{Sa}$ UNI

2020;7(1):18-24

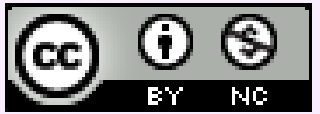

Esta obra está bajo una Licencia Creative Commons Atribución-NoComercial 4.0 Internacional.

\section{Exposición al formol y posible sintomatología en estudiantes de medicina}

\section{Exposure to formaldehyde and possible symptoms in medicine students}

\section{Exposição ao formaldeído e possíveis sintomas em estudantes de medicina}

\section{Resumen}

Introducción: El formol, una sustancia de uso común en laboratorios de morfología de la mayoría de universidades, donde se preparan soluciones sin tomar en cuenta las normas estandarizadas y principios básicos en salud y educación. El contacto causa toxicidad local originando manifestaciones principalmente respiratorias y cutáneas, como alergias. El objetivo fue identificar la sintomatología que los estudiantes perciben durante la exposición y las medidas de protección personal que utilizan como bioseguridad. Metodología: La investigación fue cuantitativo, descriptivo, transversal y retrospectivo. El muestreo fue no probabilístico, la población conformada por 684 estudiantes de diferentes universidades de Cochabamba de marzo a junio del 2019. La técnica de recolección de datos fue, una encuesta online. Resultados: Durante la semana los estudiantes concurren a los laboratorios como promedio 10 horas. El 53,9\% no recibió información sobre los efectos adversos que ocasiona. No saben la composición $50,7 \%$ y no tiene conocimiento de los efectos adversos el $61,3 \%$. La sintomatología que presentaron fue; cambios de humor $9.9 \%$, fatiga general $19.7 \%$, irritación garganta $28.8 \%$, rinorrea $29.5 \%$, fatiga ocular $30.2 \%$, lagrimeo $76.9 \%$, dolor ocular $46.3 \%$ e irritación de piel $14.4 \%$. Discusión: Se han descrito tres formas de protección cuando se emplea el formaldehido en laboratorios; protección personal, diseño adecuado de los laboratorios y suficiente y eficaz ventilación. Hay que destacar la falta de conocimiento sobre los efectos adversos y el insuficiente control de las universidades con el equipo de bioseguridad empleado por los estudiantes. Es preciso poner en práctica acciones preventivas que incluyen control y educación sobre bioseguridad en ambientes que emplean este químico.

Palabras Claves: Formaldehído, Exposición, Toxicidad, Signos y Síntomas.

\section{Summary}

Introduction: Formaldehyde, a substance commonly used in morphology laboratories in the most universities, where solutions are prepared without taking into account the standardized norms and basic principles in health and education. Contact with 
it causes local toxicity, causing mainly respiratory and skin manifestations, such as allergies. The objective was to identify the symptoms that the students perceive during its exposure and the personal protection measures that they use as biosecurity. Methodology: The research was quantitative, descriptive, cross-sectional and retrospective. The sampling was non-probability, the population was made up by 684 students from different universities in Cochabamba since March to June 2019. The data collection technique was an online survey. Results: During the week students attend the laboratories on an average of 10 hours. A 53.9\% did not receive information about the adverse effects it causes. A $50.7 \%$ do not know its composition and a $61.3 \%$ do not have knowledge about its adverse effects. The symptoms they presented were; mood swings a $9.9 \%$, general fatigue a $19.7 \%$, throat irritation a $28.8 \%$, runny nose a $29.5 \%$, eye fatigue a $30.2 \%$, tearing a $76.9 \%$, eye pain a $46.3 \%$ and skin irritation a $14.4 \%$. Discussion: Three protection forms have been described when formaldehyde is used in laboratories; personal protection, adequate laboratory design and its enough and effective ventilation. It must be stand out there is a lack of knowledge about the adverse effects and the insufficient control in the universities with the biosecurity team used by the students. Preventive actions must be put in practice, including the biosafety control and education in environments where this chemical is used.

Keywords: Formaldehyde, Exhibition, Toxicity, Signs and Symptoms.

\section{Resumo}

Introdução: Formalina, substância comumente usada em laboratórios de morfologia na maioria das universidades, onde as soluções são preparadas sem levar em consideração as normas padronizadas e os princípios básicos em saúde e educação. 0 contato causa toxicidade local causando principalmente manifestações respiratórias e cutâneas, como alergias. O objetivo foi identificar os sintomas que os alunos percebem durante a exposição e as medidas de proteção individual que utilizam como biossegurança. Metodologia: A pesquisa foi quantitativa, descritiva, transversal e retrospectiva. A amostragem de tipo não probabilística. População composta de 684 estudantes de diferentes universidades de Cochabamba no periodo de março a junho de 2019. A técnica de coleta de dados foi uma pesquisa on-line. Resultados: Durante a semana os alunos frequentam os laboratórios em média 10 horas; 53,9\%, não receberam informações sobre os efeitos adversos que causa; 50,7\%, desconhecem a composição e $61,3 \%$, desconhecem os efeitos adversos. Os sintomas que eles apresentaram foram: alterações de humor 9,9\%, fadiga geral 19,7\%, irritação na garganta $28,8 \%$, coriza $29,5 \%$, fadiga ocular $30,2 \%$, lacrimejamento $76,9 \%$, dor ocular $46,3 \%$ e irritação cutânea 14,4\%. Discussão: Três formas de proteção foram descritas quando o formaldeído é usado em laboratórios: 1. proteção pessoal, 2. projeto laboratorial adequado e 3. ventilação suficiente e eficaz. Vale destacar o desconhecimento dos efeitos adversos e o controle insuficiente das universidades com a equipe de biossegurança utilizada pelos estudantes. Ações preventivas devem ser implementadas, incluindo controle e educação sobre biossegurança em ambientes que utilizam esse produto químico.

Palavras chave: Formaldeído, Exposição, Toxicidade, Sinais e Sintomas. 


\section{Introducción}

El formaldehido es conocido como Metanal es un compuesto químico que presenta grupo funcional carbonil $(\mathrm{C}=\mathrm{O})$ y un enlace con un hidrógeno, es un gas incoloro que posee enlaces polares, su fórmula química es $\mathrm{H} 2 \mathrm{C}=\mathrm{O} 1(1,2)$.

El formol o formalina es una solución comercial de formaldehido y agua y en algunos casos metanol. Estos compuestos se caracterizan por ser irritantes en las mucosas respiratorias y conjuntivales $(1,2)$.

El formol es una sustancia de uso común en los laboratorios de Anatomía e Histología y embriología, áreas que corresponden a los departamentos de Morfología de la mayoría de universidades, donde se preparan soluciones sin tomar en cuenta las normas estandarizadas y principios básicos en salud y educación (3).

En el hombre el formaldehído causa toxicidad local originando manifestaciones clínicas principalmente respiratorias y cutáneas, como ser alergias, dermatitis localizándose principalmente en cara, cuello, superficies de flexión de los codos y los párpados (3).

Otros estudios indican irritación de la piel, dolor ocular, lagrimeo, fatiga ocular, rinorrea, irritación de garganta, fatiga general y cambios de humor (4)

Por las características; físicas, químicas, toxicológicas y condiciones de utilización, el formaldehido puede representar un riesgo en la salud de los estudiantes y los docentes (5).

La emisión de vapores de formaldehido y el contacto directo a menudo ocurren durante las prácticas de dichas materias exponiendo a los estudiantes y docentes a esta sustancia (6).

La falta de conocimiento sobre los efectos del formaldehido por parte de los estudiantes y docentes hace que no relacionen como posible causa a ciertos síntomas ni toman las medidas preventivas adecuadas (7).

Datos de estudios en América Latina reportan que las tasas de prevalencia de estos síntomas en los estudiantes están entre $50 \%$ y $71 \%$. En Bolivia no se encontró información de esta problemática, pero es evidente la importancia del uso racional del formaldehido en los laboratorios de morfología $(3,7,8)$

Cuando se utiliza formaldehido en laboratorios de anatomía se tiene identificado tres formas para protegerse, los cuales son: medidas de protección personal (uso de barbijo, guantes de látex, bata, gorra y lentes), diseño apropiado de los laboratorios (aislamiento del área de oficinas, estantes asegurados a la pared, disponibilidad de lavamanos, mesas y sillas de trabajo de materiales resistentes) y ventilación suficiente y eficaz (sistemas de extracción de vapores) $(9,10)$.

Por lo tanto, el trabajo pretende identificar la sintomatología que los estudiantes perciben durante la exposición y las medidas de protección personal que utilizan como bioseguridad recomendada.

\section{Metodología}

Cochabamba, Bolivia se ha convertido en un centro universitario importante, donde se encuentran 18 universidades entre estatales y privadas, de estas 8 ofrecen la carrera 
de medina. No se tiene el número exacto de estudiantes nacionales y extranjeros (11).

El trabajo de investigación fue cuantitativo, descriptivo, transversal y retrospectivo. El tipo de muestreo fue no probabilístico, la población estuvo conformada por 684 estudiantes de diferentes universidades que cuentan con carrera de medicina asentadas en Cochabamba de marzo a junio del 2019.

La técnica de recolección de datos aplicada fue, una encuesta online con preguntas cerradas por medio del formulario google, "Los Formularios Google te permite planificar eventos, enviar una encuesta, hacer preguntas o recopilar otros tipos de información de forma fácil y eficiente" (12).

Posteriormente los datos fueron exportados a una hoja de cálculo Excel y codificados en el programa IBM-SPSS Statistics versión 25, procediendo al análisis estadístico.

No se solicitó autorización de las universidades, porque la participación de los estudiantes fue voluntaria con el consentimiento de los mismos.

\section{Resultados}

En los meses de marzo a junio del 2019 se compartió por las redes sociales WhatsApp y Facebook la encuesta online y fue respondida por 684 estudiantes de medicina de diferentes universidades de Cochabamba, obteniéndose los siguientes resultados:

En la semana los estudiantes de medicina concurren a los anfiteatros de anatomía como promedio 10 horas para sus prácticas semanales, también acuden a estos ambientes horas adicionales: 1 hora 5.8\% $(n=40), 2$ horas 38.5\% $(n=263), 3$ horas $28.5 \%(n=195), 4$ horas $14.5 \%(n=99)$ y más 4 horas $12.7 \%(n=87)$.

El $53,9 \%$ ( $n=369)$ no recibió información sobre los efectos adversos que provoca el formaldehido.

No saben cuál es la composición del formaldehido $50,7 \%(n=390)$ y no tiene conocimiento de los efectos adversos que provoca este químico el 61,3\% ( $n=419)$.

El grafico 1 muestra el tipo de seguridad que usan los estudiantes al ingresar a los ambientes de prácticas de anatomía.

Grafico 1. Seguridad que usan los estudiantes de medicina en los anfiteatros

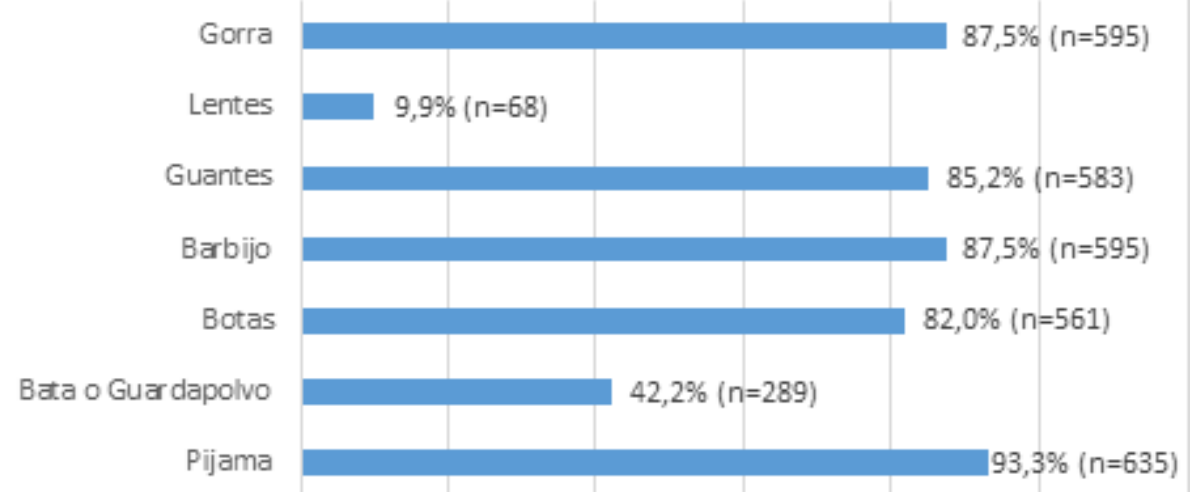

Fuente: Elaboración Propia

Los síntomas y signos que percibieron los estudiantes que están expuestos al formaldehido son: ver gráfico 2. 
Grafico 2: Síntomas y signos que los estudiantes percibieron por la exposición.

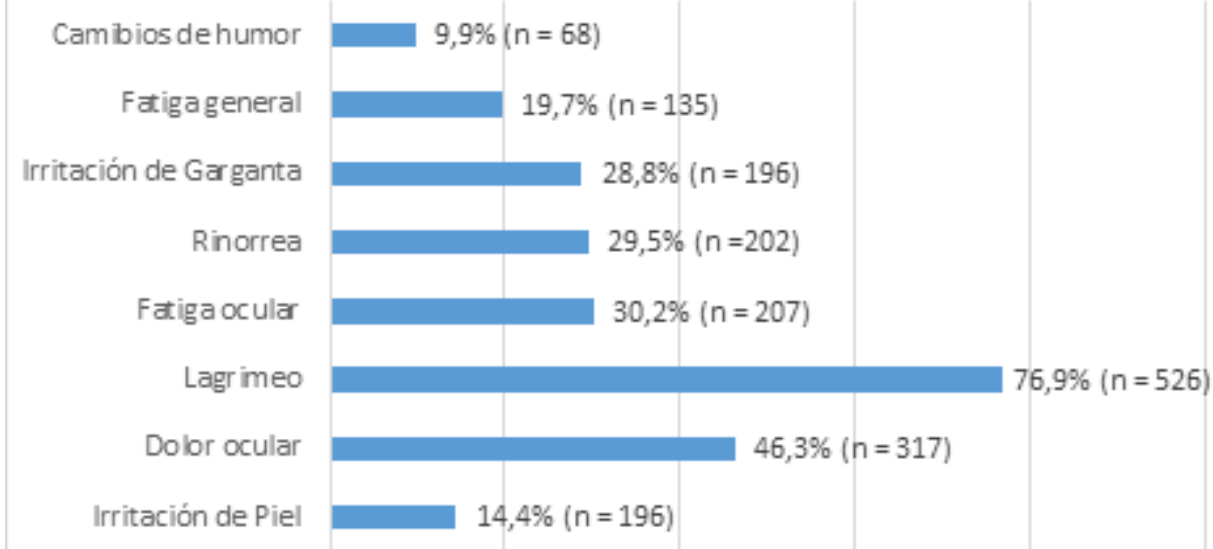

Fuente: Elaboración Propia

\section{Discusión}

Se ha destacado que pocas universidades cuentan con sistemas de ventilación local en los ambientes de prácticas donde se usa el formaldehido y la necesidad que se tomen medidas para reducir las concentraciones de dicha sustancia.

El tiempo de exposición muestra que $12.7 \%$ están expuestos a más de 14 horas semanales es decir 5 horas, tres veces por semana como máximo. Según la administración de Seguridad y Salud Ocupacional de los Estados Unidos el tiempo límite para exposición al formaldehido es de 8 horas diarias a concentraciones de 0,3 ppm; 0,37 $\mathrm{mg} / \mathrm{m} 3$. Por lo tanto el tiempo de exposición no es un riesgo aparente para la salud de los estudiantes de medicina expuestos al químico (9).

El 53,9\% no recibió información sobre los efectos adversos, $50.7 \%$ no sabe la composición y $61,3 \%$ no tiene conocimiento de los efectos adversos que provoca este químico. Es relevante incidir sobre la falta de información que los docentes de prácticas proporcionan a los estudiantes antes de ingresar a estos ambientes.

A la pregunta sobre el equipamiento de bioseguridad que emplean los estudiantes al ingresar a estos ambientes de prácticas mostraron, que no cumplen en su totalidad con lo establecido según normas internacionales; gorra $87,5 \%$, lentes $9,9 \%$, guantes $85,2 \%$, barbijo $87.5 \%$, botas $82.0 \%$, bata o guardapolvo $42.2 \%$ y pijama $93,3 \%$. No usar estas cosas sería un factor determinante para que presenten algún tipo de signos y síntomas de toxicidad (13).

La sintomatología presentada post exposición al formaldehido es: Un 9,9\% presento cambios de humor y $19 \%$ fatiga general estos datos obtenidos son superiores en relación con otros estudios donde muestra 3.3\%. No obstante, no está descrito en la literatura con claridad la relación entre el formaldehido y estos síntomas (4).

La irritación de garganta se presentó en un $28.8 \%$ y rinorrea $29,5 \%$ que se relaciona con la falta de uso de barbijo por estudiantes de medicina $12,5 \%$ que muestra nuestro trabajo, pero relacionado con otros estudios es bajo para la irritación de garganta $71,4 \%$ (14) y rinorrea $51,5 \%$.(15).

La fatiga ocular se presentó en $30,2 \%$ de los encuestados, lagrimeo $76,9 \%$ y dolor 
ocular $46,3 \%$, que también se relaciona por la falta de uso de lentes por estudiantes ya que $90,1 \%$ no lo usan, y relacionando con otros estudios estos resultados son elevados según indica para la fatiga ocular y lagrimeo 3,3\% (4).

La irritación de piel se presentó en el $14,4 \%$ de los casos esto se debe al contacto directo con el formaldehido relacionado por la falta de uso de guantes de látex que nuestro estudio muestra $14,8 \%$ y relacionando con otros estudios es bajo $25 \%$ (14).

Se han descrito tres formas de protección cuando se emplea el formaldehido en laboratorios de anatomía; protección personal, diseño adecuado de los laboratorios y suficiente y eficaz ventilación (17), nuestro estudio tiene la difidencia de no verificar los dos últimos factores descritos.

Hay que destacar la falta de conocimiento sobre los efectos adversos de la exposición al formaldehido y el insuficiente control de las universidades con el equipo de bioseguridad empleado por los estudiantes. Es preciso poner en práctica acciones preventivas que contengan un constante control y educación sobre bioseguridad en ambientes que emplean este químico.

\section{Conflictos de Intereses}

Los autores declaramos no tener conflictos de interés para el presente estudio.

\section{Referencias bibliográficas}

1. Idrobo-Avila EH, Vasquez-López JA, Vargas-Cañas R. La exposición ocupacional al formol y la nueva tabla de enfermedades laborales. Rev Salud Pública. 2017;19:382-5.

2. Brown WH, Iverson BL, Anslyn E, Foote CS. Organic Chemistry. Edición: 7. Australia ; Belmont, CA: Cengage Learning; 2013. 1312 p.

3. Parra JED, Zapata JJD. El formol su génesis, normas, aplicaciones e incidencia sobre la salud humana. Med UPB. 1999;18(1):35-45.

4. Takahashi S, Tsuji K, Fujii K, Okazaki F, Takigawa T, Ohtsuka A, et al. Prospective study of clinical symptoms and skin test reactions in medical students exposed to formaldehyde gas. J Dermatol. mayo de 2007;34(5):283-9.

5. Casas A, Muñoz A, Sitges M, Boada Mi, Munar B. Estudio longitudinal de la exposición a formaldehído en el Laboratorio de Anatomía Patológica del Hospital Universitario Son Dureta. Rev Asoc Espanola Espec En Med Trab. 2009;18:23.

6. Sarmiento Acosta N, Peinado Acevedo JS, Cadena Afanador L del P. Sintomatología causada por la exposición al formaldehído en estudiantes de medicina y sus posibles mecanismos fisiopatológicos. latreia. 2014;27(4):428-38.

7. Méndez TR de. Un caso de intoxicación crónica por formaldehído. MedULA. 1999;8(1-4):25-8.

8. Veronez DA da L, Farias ÉLP, Fraga R de, Freitas RS de, Petersen ML, Silveira JR de P. Potencial de risco para a saúde ocupacional de docentes, pesquisadores e técnicos de anatomia expostos ao formaldeído. InterfacEHS - Rev Saúde, Meio Ambient e Sustentabilidade. 2010;5(2).

9. OSHA | Administración de Seguridad y Salud Ocupacional [Internet]. [citado 7 de 
septiembre de 2019]. Disponible en: https://www.osha.gov/aboutosha

10. Skisak CM. Formaldehyde vapor exposures in anatomy laboratories. Am Ind Hyg Assoc J. 1983;44(12):948-50.

11. 18 Universidades en Cochabamba | Los Tiempos [Internet]. [citado 11 de septiembre de 2019]. Disponible en: https://www.lostiempos.com/tendencias/educacion/20180125/18-universidades-cochabamba

12. Formularios Google. Una herramienta estrella de Google. | Observatorio del Gabinete de Tele-Educación [Internet]. [citado 11 de septiembre de 2019]. Disponible en: https://blogs.upm.es/observatoriogate/2016/04/21/formularios-google-una-herramienta-estrella-de-google/

13. OSHA - Vigilancia médica - Formaldehído | Administración de Seguridad y Salud Ocupacional [Internet]. [citado 9 de septiembre de 2019]. Disponible en: https:// www.osha.gov/laws-regs/regulations/standardnumber/1910/1910.1048AppC

14. Russo T de M. Efectos tóxicos crónicos del formaldehido. MedULA. 2000;9(14):45-57.

15. Kim H, Kim YD, Cho SH. Formaldehyde exposure levels and serum antibodies to formaldehyde-human serum albumin of Korean medical students. Arch Environ Health. abril de 1999;54(2):115-8.

16. Wantke F, Focke M, Hemmer W, Tschabitscher M, Gann M, Tappler P, et al. Formaldehyde and phenol exposure during an anatomy dissection course: a possible source of IgE-mediated sensitization? Allergy. noviembre de 1996;51(11):837-41.

17. Mena Marín M, Alpízar Calvo T, Mena Umaña F. Medidas de Bioseguridad en una sala de disección de anatomía patológica. Med Leg Costa Rica. marzo de 2010;27(1):35-9. 